\title{
Muß der verunfallte Patient vor dem Notarzt geschützt werden?
}

Die Notfallmedizin ist einerseits eine der jüngsten Disziplinen in der Medizin, andererseits mit Sicherheit die Disziplin, die sich innerhalb der letzten 25 Jahre am schnellsten fortentwickelt hat. Die Etablierung der Notfallmedizin im heutigen Sinne wurde ermöglicht durch das Engagement vieler Ärzte, durch neue technische Möglichkeiten und durch die Unterstützung der politisch Verantwortlichen.

Nachdem schon recht bald deutlich wurde, wie sehr die notfallmedizinische Versorgung die Letalität zum Beispiel von unfallverletzten Patienten senken kann, wurde die Professionalisierung dieser Disziplin weiter vorangetrieben. $\mathrm{Zu}$ Anfang der Notfallmedizin gab es nur sehr grob umschriebene Richtlinien für die Qualifikation von Notärzten. Um die Qualität der präklinischen Versorgung von Unfallopfern aber auch anderer medizinischer Notfälle zu sichern, wurden spezielle Fortbildungsseminare für Notärzte eingerichtet. Erste Initiativen in diese Richtung wurden von den Notärzte-Arbeitsgemeinschaften auf Ebene der Bundesländer ergriffen. In Zusammenarbeit mit den Arbeitsgemeinschaften und der Landesärztekammern wurden vor einigen Jahren bundesweit standardisierte Richtlinien festgelegt, nach denen ein in der Notfallmedizin tätiger Arzt seine Qualifikation nachzuweisen hat.

Die Versorgung von Notfallpatienten, insbesondere von Unfallpatienten, hat sich im Laufe der Jahre, nicht zuletzt durch diese Fortbildungsveranstaltungen, erheblich verbessert. Die abnehmende Zahl von Unfallverletzungen mit Todesfolge gibt hierüber eindeutig Zeug- nis ab. Aus unfallchirurgischer Sicht waren besonders sensible Punkte die frühe Intubation und maschinelle Beatmung von Traumapatienten und das Legen einer Thoraxdrainage noch am Unfallort. Darüberhinaus wurde auch immer wieder auf die ausreichende Transfusion mit kristalloiden und kolloidalen Infusionslösungen hingewiesen. Sehr häufig wurde gerade das Legen einer Thoraxdrainage mit den übrigen an der Notfallmedizin beteiligten Disziplinen kontrovers diskutiert. Mittlerweile hat sich die Diskussion etwas gelegt und die adäquate Versorgung von Traumapatienten sollte mittlerweile als etabliert gelten.

Eine Unterversorgung von Traumapatienten haben wir im eigenen Patientenkollektiv nur noch selten beobachtet. Andererseits konnte nunmehr wiederholt festgestellt werden, daß Patienten eine maximale notfallmedizinische Therapie erhalten haben, die sie jedoch retrospektiv, nach Feststellen aller Verletzungen, nicht erhalten haben müßten. Aufgrund dieser Beobachtungen sahen wir uns zu einer Analyse der Notfallmedizinischen Therapie der uns zugewiesenen Traumapatienten veranlaßt.

Diese Analyse erhebt nicht den Anspruch, eine konkrete Qualitätsanalyse darzustellen. Letzten Endes ist die Dokumentation der präklinischen Daten hierfür oft nicht ausreichend genug. Andererseits zeigt diese Analyse, daß auch heute noch eine nicht adäquate Traumaversorgung vorkommt. Die unterversorgten Fälle mit übersehenen Verletzungen überwiegen noch bei weitem die Fälle von sozusagen „übertherapierten" Patienten. Dennoch zeigt sich hier eine Tendenz zu übertriebenen Maßnahmen am Unfallort.
Für die Arbeit der Notarztausbildenden Institutionen sollte gerade das Symptom der Übertherapie ein Alarmsignal darstellen. Bedeutet es doch, daß die Lehre von einem Trauma-Algorithmus anscheinend die differenzierte Diagnostik und individuell ausgerichtete Therapie des Traumapatienten in den Hintergrund stellt. Bei allen Entscheidungsrichtlinien, die für die notfallmedizinische Versorgung über die Jahre erarbeitet worden sind und mittlerweile fester Bestandteil der Versorgung geworden sind, sollte nicht versäumt werden, den Notarzt daraufhinzuweisen, daß jede therapeutische Maßnahme mit Bedacht durchgeführt werden muß. Nach wie vor ist es nicht nur Aufgabe des Notarztes, ein gefährdetes Leben zu retten, sondern auch weiteren Schaden von diesem abzuwenden.

Das Leitthema „Muß der verunfallte Patient vor dem Notarzt geschützt werden?", mag provokativ klingen. Die Analyse soll jedoch letzten Endes darstellen, daß trotz des hohen Standards den die notfallmedizinische Versorgung mittlerweile erreicht hat, eine ständige selbstkritische Kontrolle notwendig ist. Darüberhinaus sollten alle unfallchirurgischen Abteilungen das Leitthema zum Anlaß nehmen, ihr Engagement im Bereich der präklinischen Unfallversorgung zu überdenken und ggf. auszudehnen. Die präklinische Versorgung von Unfallverletzten ist traditionell ein chirurgisches Interesse und sollte auch in Zukunft von der Unfallchirurgie maßgeblich mitgetragen werden.

\section{A. Seekamp, H. Tscherne}

